\title{
Resolutividade da campanha de prevenção e diagnóstico precoce do câncer bucal em São Paulo, Brasil
}

\author{
José Leopoldo Ferreira Antunes, ${ }^{1}$ Tatiana Natasha Toporcov ${ }^{1}$ \\ e Victor Wünsch-Filho ${ }^{2}$
}

Como citar Antunes JLF, Toporcov TN, Wünsch-Filho V. Resolutividade da campanha de prevenção e diagnóstico precoce do câncer bucal em São Paulo, Brasil. Rev Panam Salud Publica. 2007; 21(1):30-6.

RESUMO Objetivo. Avaliar os resultados da campanha de prevenção e diagnóstico precoce do câncer bucal realizada no contexto da campanha de vacinação contra a gripe em idosos no Estado de São Paulo em 2004.

Métodos. A partir dos relatórios do Centro Técnico de Saúde Bucal da Secretaria de Estado da Saúde, foram levantados dados sobre o seguimento de pacientes encaminhados para elucidação diagnóstica de lesões de tecido mole da boca após diagnóstico visual. Foram avaliados indicadores de resolutividade da campanha (número de pessoas cujo problema foi resolvido no nível básico de atenção à saúde ou em serviços de referência; e pessoas com diagnóstico confirmado de câncer bucal) e de falta de resolutividade (não comparecimento dos encaminhados na unidade de referência; incapacidade do nível básico de atenção à saúde de resolver o problema e não identificação de serviços de referência com capacidade para resolver o problema; e pessoas sem informação quanto ao seguimento).

Resultados. Em 2004, foram examinadas 238087 pessoas de 60 anos ou mais, correspondendo a 6,8\% da população do Estado nessa faixa etária (3 494555 habitantes). A campanha foi realizada em 23 das 24 direções regionais de saúde, porém apenas oito regiões registraram informações sobre seguimento. Das 5280 pessoas encaminhadas para elucidação diagnóstica de lesões em tecidos moles da boca nessas oito regiões, 60,5\% tiveram o seu problema resolvido e 0,5\% (26 casos) tiveram diagnóstico confirmado de câncer bucal; 22,5\% não completaram o diagnóstico. Para 16,5\% dos encaminhamentos, não houve informação disponível quanto ao seguimento e desfechos.

Conclusões. Considerando-se a falta de monitoramento dos resultados na maior parte do Estado e a elevada proporção de pacientes cujo problema de lesão em tecido mole da boca não pôde ser resolvido, a campanha mostrou-se ineficaz. É preciso avaliar a conveniência de realizar a campanha nos próximos anos.

Palavras chave Estudos de avaliação, neoplasias bucais, programas de rastreamento, Brasil.

1 Universidade de São Paulo (USP), Faculdade de Odontologia, São Paulo (SP), Brasil. Enviar correspondência para José Leopoldo Ferreira Antunes no seguinte endereço: Avenida Professor Lineu Prestes 2227, CEP 05508-900, São Paulo, SP, Brasil. Fone: +55-11-3091-7877; fax: +55-11-3091-7874. E-mail: leopoldo@usp.br

2 USP, Faculdade de Saúde Pública.
Na Cidade de São Paulo, nas duas últimas décadas, a mortalidade por câncer bucal manteve-se estacionária, porém elevada (1). No Estado, a incidência desse tipo de câncer também é elevada em relação a outros contextos urbanos no país e no exterior, sendo o sofrível perfil de sobrevivência dos pacientes associado ao retardo no diagnóstico (2). Em 2005, o Instituto Nacional de Câncer estimou, para o Estado de São Paulo, a 
incidência de 17,77 casos de câncer de cavidade oral para homens e 5,59 para mulheres (taxas brutas por 100000 habitantes) (3). A partir de 2000, a mortalidade por câncer de boca (incluindo faringe) tem sido em média 6,95 para homens e 1,20 para mulheres (taxas brutas por 100000 habitantes) residentes no Estado. Quando considerada apenas a faixa etária de 60 anos ou mais, esses números sobem para 40,77 e 7,74, respectivamente (4).

No Brasil, a prevenção primária do câncer de boca consiste fundamentalmente em programas e medidas de combate ao consumo de tabaco e álcool, num esforço integrado de promoção da saúde que visa à redução de vários outros agravos. O exame visual da boca para detecção precoce de lesões cancerizáveis e tumores não sintomáticos é uma estratégia de prevenção secundária intuitiva e atraente, a partir da qual se espera viabilizar o diagnóstico da doença em seus estágios iniciais e, assim, possibilitar um melhor prognóstico por meio da pronta e efetiva intervenção terapêutica. Com o objetivo de integrar os dois níveis de prevenção, foi implementada, em 2001, no Estado de São Paulo, uma campanha anual de prevenção (medidas educativas e de orientação) e diagnóstico precoce (visual) de lesões suspeitas de câncer bucal.

Embora o diagnóstico de tumores de boca em estágio inicial implique um melhor prognóstico para os pacientes (5), as evidências epidemiológicas não têm dado suporte a iniciativas de rastreamento de câncer bucal, uma vez que os estudos sobre medidas terapêuticas associadas a esses programas não permitiram conclusões definitivas a respeito de benefícios quantificáveis $(6,7)$. Seria ineficaz indicar procedimentos de rastreamento para o câncer bucal, principalmente em contextos com recursos escassos para as ações de saúde (8). Não obstante, os organismos de saúde do Canadá e dos Estados Unidos consideram recomendável a realização periódica de exames bucais para pessoas com mais de 60 anos, fumantes e com perfil de consumo elevado de bebidas alcoólicas $(6,7,9)$. Um recente estudo de base populacional para avaliar o impacto do exame visual da cavidade bucal sobre o câncer oral na Índia mostrou que esse tipo de rastreamento pode, efetivamente, reduzir a mortalidade em grupos de alto risco (10).

O estudo de resolutividade dos esforços de rastreamento do câncer bucal é complexo e envolve múltiplas dimensões. $\mathrm{O}$ primeiro e principal objetivo do rastreamento é identificar casos suspeitos e possibilitar a rápida aplicação de recursos laboratoriais visando a confirmar ou rejeitar a suspeita diagnóstica. Portanto, a avaliação dessas campanhas deve incluir a verificação quanto ao seguimento dos pacientes encaminhados para a realização de procedimentos diagnósticos e tratamento imediato ou, afastada a suspeita de câncer, tratamento adequado das lesões de tecido mole da boca.

Considerando a necessidade de conscientizar a população sobre os riscos relacionados ao câncer bucal e de efetivar uma estratégia de diagnóstico precoce de casos da doença, a Secretaria de Estado da Saúde de São Paulo implementou, em 2001, a participação de cirurgiõesdentistas nas campanhas anuais de vacinação de idosos contra a gripe. Desse modo, os profissionais de saúde têm acesso a um contingente da população em faixa etária de risco mais elevado para a doença. Nesse contato, os profissionais de saúde buscam prover orientações quanto à prevenção e redução do consumo de tabaco e bebidas alcoólicas e realizar exames bucais para a detecção de lesões de tecido mole da boca, além de avaliar a condição e a necessidade de próteses dentárias.

A avaliação da resolutividade das campanhas realizadas em São Paulo é dificultada pela necessidade de recuperar informações em diferentes níveis de atenção, pelo elevado número de profissionais e usuários envolvidos e pela insuficiente capacitação da campanha em efetivar o encaminhamento e definir o diagnóstico para os indivíduos classificados como suspeitos. Essas considerações motivaram a presente comunicação, cujo objetivo é relatar os resultados contabilizados pela campanha de prevenção e diagnóstico precoce do câncer bucal no Estado de São Paulo em 2004 e discutir suas conseqüências.

\section{MÉTODOS}

Este é um estudo descritivo dos resultados da campanha de prevenção e diagnóstico precoce do câncer bucal realizada no Estado de São Paulo em abril de 2004, sob a coordenação do Centro Técnico de Saúde Bucal da Secretaria de Estado da Saúde. Embora seja realizado anualmente por ocasião da campanha de vacinação contra a gripe, o rastreamento de câncer bucal pelo exame visual da mucosa não é uma medida incorporada à rotina dos serviços de saúde no Estado de São Paulo. Nesse sentido, não há protocolos formais pré-definidos para orientar a sua realização ou avaliar os seus resultados (11).

O procedimento adotado na campanha aqui analisada consistiu no preenchimento de questionário de identificação pessoal, incluindo informações quanto a hábitos de alcoolismo e tabagismo; exame odontológico de cárie e da condição e necessidade de prótese dentária, segundo metodologia padronizada internacionalmente pela Organização Mundial da Saúde (OMS) (12); e exame visual para detecção de lesões em lábios, língua, cavidade oral, amígdala e orofaringe (13). Os cirurgiões-dentistas participantes da campanha distribuíam um folheto explicativo e prestavam informações sobre a prevenção de câncer bucal, reiterando a necessidade de elucidação diagnóstica e tratamento das lesões de tecido mole da boca, informando inclusive que algumas dessas lesões podem evoluir para neoplasias.

As pessoas que apresentaram lesões de tecidos moles da boca foram encaminhadas para elucidação diagnóstica nas unidades de atendimento básico em saúde, onde receberam tratamento ou foram 
encaminhadas para serviços de referência. Os dentistas que realizavam a triagem eram instruídos a não revelar qualquer suspeita de malignidade, mesmo porque o registro efetuado seria utilizado para busca ativa em caso de não comparecimento do examinado na unidade de referência. Como o objetivo do programa é encaminhar os pacientes para elucidação diagnóstica, o presente estudo de resolutividade se deteve na avaliação dos encaminhamentos efetuados em 2004, não tendo se restringido apenas aos indivíduos considerados como portadores de lesões suspeitas de malignidade. Esse foco também foi escolhido em função da falta de informações completas, que impediu a aplicação de recursos convencionais da epidemiologia para a análise de sensibilidade e especificidade, ou seja, o reconhecimento de falso-positivos e falso-negativos no diagnóstico do câncer de boca.

Apesar das dificuldades para monitorar os resultados da campanha, foram emitidos relatórios (13-15) consolidando informações sobre a cobertura do programa nas campanhas realizadas de 2001 a 2004. Esses relatórios informaram o número: de pessoas examinadas; de pessoas que apresentaram lesões de tecidos moles da boca; e de pessoas com lesões avaliadas pelo profissional de saúde como suspeitas de malignidade.

Em 2004, foi emitido um relatório adicional (16), com informações mais detalhadas sobre o seguimento das pessoas encaminhadas para elucidação diagnóstica em 207 cidades de oito direções regionais de saúde (DIR): DIR V, Osasco (15 cidades); VI, Araçatuba (40 cidades); VIII, Assis (16 cidades); XVII, Registro (5 cidades); XVIII, Ribeirão Preto (25 cidades); XIX, Santos (7 cidades); XX, São João da Boa Vista (18 cidades); e XXII, São José do Rio Preto (81 cidades).

Com base nesses relatórios, foram selecionados dados simplificados que refletissem a cobertura e os resultados do programa de rastreamento do câncer de boca no Estado de São Paulo. Como indicadores de resolutividade da campanha, foram levantados os números e as porcentagens de pessoas que: a) tiveram seu problema resolvido no nível básico de atenção à saúde; b) tiveram seu problema resolvido após encaminhamento para serviços de referência; e c) tiveram diagnóstico confirmado de câncer bucal. A falta de resolutividade foi avaliada por meio dos números e porcentagens de: a) pessoas que não atenderam à solicitação de encaminhamento; b) pessoas cujo problema não foi resolvido no nível básico de atenção à saúde nem tampouco puderam ser encaminhadas para serviços de referência; e c) pessoas sem informação quanto ao seguimento. Esses dados foram extraídos dos relatórios administrativos das regionais de saúde encaminhados ao nível central de coordenação da campanha, com base em registros das unidades de saúde envolvidas. Não foram realizados inquéritos populacionais para avaliar a cobertura e o impacto da campanha.

\section{RESULTADOS}

A parcela da população com 60 anos ou mais no Estado de São Paulo que participou do exame clínico da boca durante as campanhas de vacinação contra a gripe ampliou-se de $4,1 \%$ em
2001, primeiro ano do programa, para 8,7\% em 2004 (figura 1). Em 2004, foram realizados 238087 exames bucais em pessoas de 60 anos ou mais residentes em 490 cidades do Estado de São Paulo, correspondendo a 6,8\% da população nessa faixa etária (estimada em 3494555 habitantes) e a $8,7 \%$ do total de 2727952 pessoas vacinadas contra a gripe (tabela 1 ). A campanha de saúde bucal só não foi realizada em uma região de saúde do Estado, a DIR XI, Botucatu.

Do total de examinados em 2004, 8,5\% foram encaminhados para elucidação diagnóstica e $0,9 \%$ foram considerados portadores de lesões em tecidos moles da boca suspeitas de malignidade (tabela 1). Quinze DIR não informaram dados da campanha. Conseqüentemente, os dados analisados restringem-se a oito regionais de saúde (DIR V, Osasco; VI, Araçatuba; VIII, Assis; XVII, Registro; XVIII, Ribeirão Preto; XIX, Santos; XX, São João da Boa Vista; e XXII, São José do Rio Preto). Essas oito regionais englobam 207 municípios nos quais foram examinados, em 2004, 70615 indivíduos. A cobertura em relação ao total da população foi de $8,7 \%$ e, em relação ao total de vacinados, de $10,6 \%$. Foram encaminhadas para elucidação diagnóstica 5280 pessoas, tendo sido identificadas 511 lesões suspeitas de malignidade.

FIGURA 1. Cobertura da vacinação contra a gripe e do exame bucal em vacinados com 60 anos ou mais, Estado de São Paulo, 2001 a 2004

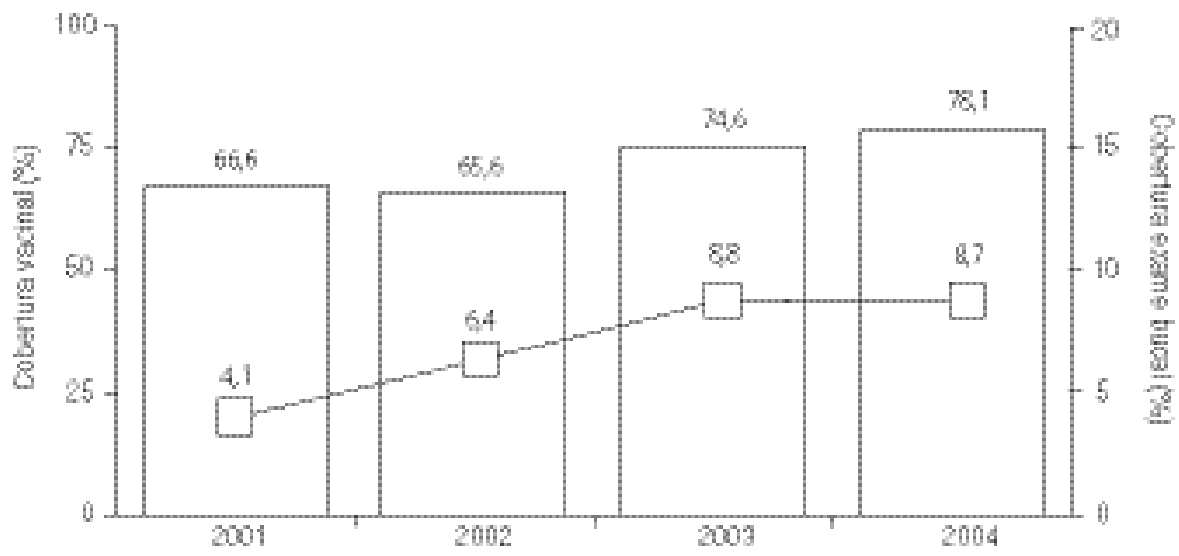

Fonte: Estado de São Paulo, Secretaria de Estado da Saúde, Coordenadoria de Planejamento em Saúde, Centro Técnico de Saúde Bucal (13-16). 
TABELA 1. Características das campanhas de prevenção e diagnóstico precoce de câncer bucal, Estado de São Paulo, 2001 a 2004 ${ }^{a}$

\begin{tabular}{lrrrr}
\hline \multicolumn{1}{c}{ Característica } & 2001 & 2002 & 2003 & 2004 \\
\hline Cidades participantes & 334 & 434 & 512 & 490 \\
Total de indivíduos examinados & 90886 & 142774 & 226540 & 238087 \\
Encaminhamentos para elucidação diagnóstica & 25185 & 13801 & 18059 & 20270 \\
Lesões suspeitas de malignidade & $\mathrm{ND}^{\mathrm{b}}$ & 2136 & 2265 & 2065 \\
\hline
\end{tabular}

Fonte: Estado de São Paulo, Secretaria de Estado da Saúde, Coordenadoria de Planejamento em Saúde, Centro Técnico de Saúde Bucal (13-15).

a Exame visual em pessoas de 60 anos ou mais que participaram da campanha de vacinação contra a gripe.

${ }^{\mathrm{b}} \mathrm{ND}$ = informação não disponível.

TABELA 2. Indicadores de resolutividade da campanha de prevenção e diagnóstico precoce de câncer bucal, Estado de São Paulo, 2004ª

\begin{tabular}{lrr}
\hline \multicolumn{1}{c}{ Indicadores } & No. & $\%$ \\
\hline Resolutividade & & \\
Pessoas com problema resolvido na atenção básica & 2418 & 45,8 \\
Pessoas com problema resolvido em serviços de referência & 778 & 14,7 \\
Pessoas com diagnóstico de câncer bucal confirmado & 26 & 0,5 \\
$\quad$ Total & 3222 & 61,0 \\
& & \\
Falta de resolutividade & 185 & 3,5 \\
Pessoas que não atenderam ao encaminhamento, com justificativa & 885 & 16,8 \\
Pessoas que não atenderam ao encaminhamento, sem justificativa & 36 & 0,7 \\
Pessoas para as quais não se localizou serviço de referência para & 77 & 1,5 \\
$\quad$ resolução do problema & 875 & 16,5 \\
Pessoas para as quais outros motivos impediram a resolução do problema & 2058 & 39,0 \\
Pessoas sem informação quanto ao seguimento & 5280 & 100,0 \\
$\quad$ Total & & \\
Total geral & & \\
\hline
\end{tabular}

Fonte: Estado de São Paulo, Secretaria de Estado da Saúde, Coordenadoria de Planejamento em Saúde, Centro Técnico de Saúde Bucal (16).

${ }^{a}$ Exame visual em pessoas de 60 anos ou mais que participaram da campanha de vacinação contra a gripe.

A tabela 2 mostra os indicadores de resolutividade da campanha de 2004. Das pessoas com seguimento conclusivo e resolução do problema de lesão em tecido mole da boca, identificou-se um primeiro grupo $(45,8 \%)$, comportando indivíduos cujo problema foi resolvido no nível básico de atenção à saúde, seja por meio de biópsia, tratamento cirúrgico ou medicamentoso, conduta expectante, remoção de fatores traumáticos ou outras intervenções. Um segundo grupo, composto por $14,7 \%$ dos indivíduos encaminhados para elucidação diagnóstica, foi acolhido pelos serviços de referência e, supostamente, teve encaminhamento adequado. Finalmente, 26 pessoas tiveram diagnóstico confirmado de câncer bucal nas oito DIR monitoradas, correspondendo a $0,5 \%$ dos indivíduos encaminhados para elucidação diagnóstica. De acordo com as informações disponíveis, a metade teve o diagnóstico confirmado no nível de atenção básica e os demais em serviços de referência. Apenas um desses pacientes não teve o tratamento iniciado em 2004.

Com base nesses dados, o Centro Técnico de Saúde Bucal da Secretaria de Estado da Saúde estimou que, de 238087 exames bucais realizados no Estado durante a campanha de 2004, 92 casos de câncer de boca podem ter sido diagnosticados, embora nenhuma inferência possa ser feita sobre o estadiamento dos tumores detectados. A Secretaria presumiu ainda que 88 dos casos rastreados de câncer de boca $(96 \%)$ teriam iniciado o tratamento em 2004. Um total de 1070 pessoas não seguiu a orientação de encaminhamento para elucidação diagnóstica. Dentre os que atenderam o encaminhamento, não foi registrado o seguimento de 875 casos. Para 36 pessoas, não se conseguiu identificar serviços de referência, e para 77 pessoas outros motivos teriam impedido a resolução do problema (tabela 2).

\section{DISCUSSÃO}

Realizadas com regularidade desde o final dos anos 1990, as campanhas nacionais de vacinação contra a gripe foram inicialmente direcionadas aos indivíduos com 65 anos ou mais. A partir de 2000, a idade de corte para a vacinação anual foi reduzida para 60 anos (17). Nos últimos anos, a cobertura vacinal ultrapassou a marca de $80 \%$ da população-alvo em todo o país $(17,18)$.

A valorização da campanha de vacinação contra a gripe facilitou a implantação da campanha de prevenção e diagnóstico precoce de câncer bucal no Estado de São Paulo. A integração das duas campanhas pode ser entendida como um esforço suplementar do serviço de saúde, aproveitando um momento privilegiado de interação com a população de 60 anos ou mais e os recursos físicos e humanos já alocados na rede estadual de saúde.

A lógica do rastreamento em câncer é identificar casos ainda em fase inicial, para os quais, supostamente, as intervenções terapêuticas podem ser benéficas, tanto em termos de sobrevida quanto de qualidade de vida. As principais restrições ao rastreamento de câncer dizem respeito à reduzida proporção de pessoas testadas que são efetivamente beneficiadas, aos interesses comerciais associados, aos efeitos adversos relacionados ao baixo valor preditivo positivo dos recursos diagnósticos e à falta de evidências suficientes de efetividade. Além disso, a 
implementação do diagnóstico precoce e da terapêutica apropriada não consegue, em muitos casos, evitar a progressão letal da doença (19).

No que diz respeito ao rastreamento do câncer bucal, ao menos parte dessas críticas estão minimizadas. Por envolver apenas a realização do exame visual da boca, não há custos vultosos associados a exames laboratoriais. Sendo dirigido ao diagnóstico de lesões em tecidos moles, o exame clínico da boca seleciona e encaminha os pacientes para a elucidação diagnóstica e, quando confirmada a lesão maligna, tratamento. Além disso, o número de dentistas contratados pelo serviço público no Estado de São Paulo seria compatível com a ampliação desse programa nos próximos anos, e o período relativamente curto (2 semanas) das campanhas de vacinação pode representar uma facilidade operacional para sua implementação. Mesmo que o rastreamento de câncer bucal não fosse realizado, as demais tarefas (educação em saúde, exame de próteses e tratamento de lesões em tecidos moles da boca) poderiam justificar a integração do exame bucal nas campanhas de vacinação. No contexto internacional, as campanhas de vacinação contra a gripe têm sido descritas como uma das iniciativas em saúde com menor diferencial de utilização entre os estratos sociais (20). Portanto, o período anual em que são realizadas consiste em oportunidade ímpar para a interação com estratos de população menos acessíveis a outras iniciativas de promoção da saúde.

Mesmo com esses potenciais aspectos positivos, os resultados deste estudo revelaram vários problemas estruturais da campanha de prevenção e diagnóstico precoce do câncer bucal no Estado de São Paulo. Um primeiro aspecto diz respeito à insuficiente abrangência dos dados obtidos. Apenas oito regionais de saúde elaboraram relatórios sobre o seguimento dos casos, correspondendo a pouco mais de um quarto de todos os encaminhamentos. Além disso, os relatórios apresentados não seguiram um padrão uniforme e continham inconsistências identificadas pelo nível central de gerência do programa (16). Embora o esforço de avaliação tenha disponibilizado informações importantes e valiosas, inclusive para o presente estudo, é necessário considerar uma sistemática mais rigorosa de registro dos dados. Campanhas de rastreamento de câncer de boca focalizadas em grupos de maior risco têm sido defendidas, desde que haja uma adequada provisão de recursos humanos com o intuito de propiciar dados para a avaliação de sua resolutividade (21). Logo, avaliações não abrangentes e pouco criteriosas subtraem aos programas de rastreamento de câncer uma de suas mais importantes finalidades.

Outros indicadores de falta de resolutividade da campanha aqui analisada são evidentes. Em uma proporção considerável $(16,8 \%)$, os encaminhamentos para elucidação diagnóstica não foram seguidos pela população, que sequer justificou o não comparecimento. Em outra elevada proporção de encaminhamentos $(16,5 \%)$, não foi possível esclarecer o desfecho do seguimento, por falta de informações. Para 113 pessoas que atenderam ao encaminhamento sugerido pela equipe de saúde bucal durante a campanha, não foi possível a resolução do problema, em parte pela não localização de um serviço de referência capacitado para receber a demanda (36 pessoas). Observou-se que tais ocorrências estiveram concentradas nas DIR VIII e XXII (Assis e São José do Rio Preto), indicando uma dificuldade regional no estabelecimento de referências efetivas para níveis hierárquicos superiores de assistência médica. Tais observações caracterizam falhas básicas no programa de rastreamento de câncer bucal aqui examinado e são fontes de preocupação, pois sugerem omissões no processo de planejamento da campanha.

Desses fatos, depreende-se que a baixa resolutividade da campanha decorreu não apenas de aspectos específicos relacionados aos procedimentos de rastreamento, mas também da frágil organização dos serviços de saúde nessas regiões, e reflete falhas no sistema de referência e contra-referência. Obviamente, essas são dificuldades que deveriam ter sido antevistas na fase de planejamento da campanha.

Outro aspecto que não foi possível avaliar por meio dos dados disponíveis diz respeito à forma de comunicação do problema identificado às pessoas com lesões que foram consideradas suspeitas de malignidade. Como o objetivo da inspeção visual é o diagnóstico de lesões em tecidos moles da boca, não cabe ao examinador levantar qualquer suspeita de malignidade para o indivíduo examinado antes da adequada elucidação diagnóstica. Considerando-se o registro de diversas falhas no planejamento da campanha, pode-se inferir a possibilidade de um treinamento insuficiente dos profissionais envolvidos. Assim, apesar de as diretrizes da campanha estarem alicerçadas em princípios éticos bem definidos, não se pode descartar a hipótese de que pessoas com suspeita de lesão maligna tenham sido comunicadas desse fato pelos profissionais avaliadores, pondo em risco de dano psicológico ao menos alguns dos participantes.

$\mathrm{O}$ adequado treinamento dos profissionais de saúde é uma etapa fundamental dos programas de rastreamento de câncer, e pode evitar sofrimento e apreensão injustificados. Esse treinamento exige a elaboração de um manual de procedimentos, com fotografias coloridas das lesões a serem identificadas, explicitação de mucosa normal, de lesões benignas, de lesões précancerosas e de câncer. Além disso, é necessária a definição dos procedimentos do exame clínico, com a exploração sistemática de todas as regiões da boca.

Iniciativas de rastreamento não são recomendadas para o câncer bucal. Uma recente revisão sistemática (22) indicou apenas um estudo randômico controlado de avaliação do rastreamento visual de câncer bucal. Os autores consideraram insuficiente o conhecimento acumulado quanto ao potencial efeito benéfico do rastreamento visual em termos de sobrevida e qualidade de vida dos pacientes ou da prevenção de complicações como comprometimento linfático e ocorrência de metástases à distância. Todavia, um ensaio populacional realizado num contexto de elevada prevalência da doença (Índia), avaliando rastreamento de câncer oral com base na inspeção visual da boca, revelou redução da mortalidade por esse câncer entre indivíduos de grupos de alto risco, como fumantes e consumidores de álcool (10).

Estima-se que a mortalidade por câncer bucal possa ser reduzida pelo esforço em 
identificar lesões precoces em segmentos de população com risco mais elevado e efetivo tratamento dessas lesões malignas (10, 21). Entretanto, os dados aqui analisados indicaram insuficiente resolutividade do rastreamento de câncer bucal associado à campanha de vacinação de idosos no Estado de São Paulo. Compreende-se, contudo, os motivos de seu forte apelo entre os profissionais de saúde e os gestores dos programas de saúde bucal, pois o exame visual da boca apresenta facilidades naturais para a abordagem clínica do cirurgião-dentista e não induz desconforto para o examinado.

Sem dúvida, há facilidades operacionais para a ampliação dessa campanha de rastreamento de câncer oral no Estado de São Paulo, e os serviços de saúde dispõem de infra-estrutura já instalada para viabilizar um controle mais estrito de seus resultados. Porém, muito esforço ainda será necessário na implementação de um rigoroso planejamento das ações, como o adequado treinamento dos cirurgiõesdentistas envolvidos no programa, a clara definição de um sistema de referência efetivo, com capacidade para atender à demanda encaminhada, e a organização de um sistema de informação eficiente, que permita informar os dados finais de todas as regiões de saúde envolvidas no programa. Sem a garantia dessas premissas, essas campanhas de rastreamento em câncer oral não passam de iniciativas bem intencionadas, mas ineficazes para reverter o impacto da doença na comunidade.

\section{REFERÊNCIAS}

1. Antunes JL, Biazevic MG, de Araujo ME, Tomita NE, Chinellato LE, Narvai PC. Trends and spatial distribution of oral cancer mortality in São Paulo, Brazil, 1980-1998. Oral Oncol. 2001;37(4):345-50.

2. Wünsch-Filho V. The epidemiology of oral and pharynx cancer in Brazil. Oral Oncol. 2002;38(8):737-46.

3. Brasil, Ministério da Saúde, Secretaria de Atenção à Saúde, Instituto Nacional de Câncer, Coordenação de Prevenção e Vigilância. Estimativa 2005: incidência de câncer no Brasil. Rio de Janeiro: INCA; 2004.

4. Brasil, Ministério da Saúde, DATASUS. Informações de saúde. Disponível em: http:// tabnet.datasus.gov.br/cgi/deftohtm.exe?sim/ cnv/ obtSP.def. Acessado em agosto de 2006.

5. Carvalho AL, Singh B, Spiro RH, Kowalski LP, Shah JP. Cancer of the oral cavity: a comparison between institutions in a developing and a developed nation. Head Neck. 2004; 26(1):31-8.

6. Hawkins RJ, Wang EE, Leake JL. Preventive health care, 1999 update: prevention of oral cancer mortality. The Canadian Task Force on Preventive Health Care. J Can Dent Assoc. 1999;65(11):617.

7. Fisher M, Eckhart C, eds. Guide to clinical preventive services: an assessment of the effectiveness of 169 interventions. Report of the U.S. Preventive Services Task Force. 2nd ed. Baltimore, Maryland: Williams \& Wilkins; 1996.

8. Lavelle CL, Scully C. Criteria to rationalize population screening to control oral cancer. Oral Oncol. 2005;41(1):11-6.

9. U.S. Department of Health and Human Services. Oral Health in America: a report of the surgeon general. Rockville, MD: U.S. Department of Health and Human Services, National Institute of Dental and Craniofacial Research, National Institutes of Health; 2000.
10. Sankaranarayanan R, Ramadas $K$, Thomas G, Muwonge R, Thara S, Mathew B, et al. Oral Cancer Screening Study Group. Effect of screening on oral cancer mortality in Kerala, India: a cluster-randomized controlled trial. Lancet. 2005;365(9475):1927-33.

11. Zoorob R, Anderson R, Cefalu C, Sidani M. Cancer screening guidelines. Am Fam Physician. 2001;63(6):1101-12.

12. World Health Organization. Oral health surveys: basic methods. 4th ed. Geneva: World Health Organization; 1997.

13. Estado de São Paulo, Secretaria de Estado da Saúde, Coordenadoria de Planejamento em Saúde, Centro Técnico de Saúde Bucal. Prevenção e diagnóstico precoce do câncer bucal [relatório final]. Projeto exame bucal e ações educativas durante a vacinação dos idosos, abril de 2004. São Paulo: Secretaria de Estado da Saúde; 2004.

14. Estado de São Paulo, Secretaria de Estado da Saúde, Coordenadoria de Planejamento em Saúde, Centro Técnico de Saúde Bucal. Prevenção e diagnóstico precoce do câncer bucal [relatório final]. Projeto impacto: exame bucal e ações educativas durante o período de $24 / 04$ a 05/05/2001-vacinação dos idosos. Projeto exame bucal e ações educativas durante a vacinação dos idosos, em abril de 2002_-versão 2002. São Paulo: Secretaria de Estado da Saúde; 2002

15. Estado de São Paulo, Secretaria de Estado da Saúde, Coordenadoria de Planejamento em Saúde, Centro Técnico de Saúde Bucal. Prevenção e diagnóstico precoce do câncer bucal [relatório final]. Projeto exame bucal e ações educativas durante a vacinação dos idosos, em abril de 2003. São Paulo: Secretaria de Estado da Saúde; 2004
Todos esses aspectos devem ser considerados na discussão sobre a resolutividade da campanha de prevenção e diagnóstico precoce do câncer bucal em idosos e sua possível extensão nos próximos anos. Os resultados aqui informados são parte da reflexão necessária para que a realização dessa campanha, assim como a proposição de sua ampliação, não se fundamentem apenas em uma expressão voluntarista e na falsa percepção de que qualquer atividade de saúde pública é válida para o controle do câncer bucal na população.

Agradecimentos. O presente estudo foi apoiado pelo Conselho Nacional de Desenvolvimento Científico e Tecnológico (CNPq), processo no. 302541/ 2004-7
16. Estado de São Paulo, Secretaria de Estado da Saúde, Coordenadoria de Planejamento em Saúde, Centro Técnico de Saúde Bucal. Relatório preliminar da $2^{a}$ fase da campanha de prevenção e diagnóstico precoce de câncer bucal-2004. São Paulo: Secretaria de Estado da Saúde; 2004.

17. Forleo-Neto E, Halker E, Santos VJ, Paiva TM Toniolo-Neto J. Influenza. Rev Soc Bras Med Trop. 2003;36(2):267-74.

18. Barros FR, Daufenbach LZ, Vicente MG, Soares MS, Siqueira M, Carmo EH. O desafio da influenza: epidemiologia e organização da vigilância no Brasil. Boletim eletrônico epidemiológico. 2004;4(1):1-7. Disponível em: http://dtr2001. saude.gov.br/svs/pub/boletim_eletronico_epi/ Boletim_eletronico_01_04.pdf. Acessado em agosto de 2006

19. Eluf-Neto J, Wünsch-Filho V. Screening faz bem à saúde? Rev Ass Med Brasil. 2000;46(4): 310-1.

20. Lorant V, Boland B, Humblet P, Deliege D. Equity in prevention and health care. $\mathrm{J}$ Epidemiol Community Health. 2002;56(7):510-6.

21. Warnakulasuriya KA, Johnson NW. Strengths and weaknesses of screening programmes for oral malignancies and potentially malignant lesions. Eur J Cancer Prev. 1996;5(2):93-8.

22. Kujan O, Glenny AM, Duxbury J, Thakker N, Sloan P. Evaluation of screening strategies for improving oral cancer mortality: a Cochrane systematic review. J Dent Educ. 2005;69(2): $255-65$.

Manuscrito recebido em 23 de fevereiro de 2006. Aceito em versão revisada 15 de agosto de 2006. 
ABSTRACT Objective. To evaluate the results of the 2004 oral cancer prevention and early diagnosis program carried out in conjunction with the annual flu vaccination campaign for the elderly in the state of São Paulo, Brazil.

The effectiveness of the oral cancer prevention and early diagnosis program in São Paulo, Brazil

\section{Key words Evaluation studies, mouth neoplasms, mass screening, Brazil.}

Methods. Data concerning the follow-up of patients referred for diagnostic investigation of soft tissue lesions identified on visual inspection were collected from the reports issued by the State Health Department/Oral Health Technical Center. The following two characteristics were evaluated as indicators of the program's effectiveness: (1) the number of people whose problem was resolved at the primary care level or in referral services and (2) the number of persons with a confirmed diagnosis of oral cancer. The following indicators were used to assess program ineffectiveness: (1) patients not appearing at the referral unit, (2) inability of the primary care services to resolve the problem, and (3) the absence of follow-up information on patients.

Results. In 2004, 238087 people $\geq 60$ years old were examined, corresponding to $6.8 \%$ of the state population in this age group ( 3494555 people). The program was carried out in 23 of the state's 24 health regions. However, only 8 of the regions recorded follow-up information. Of the 5280 people in the 8 regions who were referred for diagnostic investigation of soft tissue lesions, $60.5 \%$ had their problem resolved, $0.5 \%$ (26 cases) had a confirmed diagnosis of oral cancer, and $22.5 \%$ did not have the diagnostic investigation completed. For $16.5 \%$ of the cases referred for further study, there was no information available concerning follow-up and outcomes.

Conclusions. The oral cancer prevention and early diagnosis program was ineffective, given the lack of monitoring of results in most parts of the state of São Paulo, and the high proportion of patients whose soft tissue lesion was not resolved. The usefulness of continuing the program in future years needs to be evaluated.

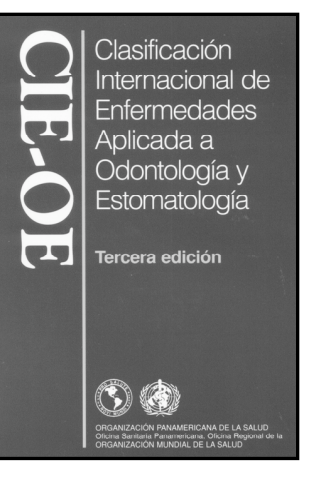

1996, 238 pp.,

ISBN 9275315620

Código: PC 562,

Precio: US\$ 22.00 en

América Latina y el

Caribe/ US\$ 32.00 en el

resto del mundo

\section{Clasificación Internacional de Enfermedades Aplicada a Odontología y Estomatología (CIE-OE), 3. ${ }^{a}$ ed.}

Esta tercera edición de la Clasificación Internacional de Enfermedades Aplicada a Odontología y Estomatología (CIE-OE) tiene por objeto proveer bases prácticas y convenientes para codificar y clasificar datos relativos a los trastornos bucales y dentarios. La CIE-OE se deriva directamente de la Décima Revisión de la Clasificación Internacional de Enfermedades (CIE-10), y ha sido concebida como parte de la "familia"de clasificaciones de enfermedades y problemas relacionados con la salud.

En la CIE-OE se incluyen todas las enfermedades y trastornos que se presentan en la cavidad bucal y estructuras adyacentes, tienen manifestaciones en ellas o están aso-ciadas con las mismas. La mayoría de las clasificaciones provistas por la CIE-10 han sido subdivididas y expandidas para incluir un quinto carácter. El propósito de la inclusión de este quinto carácter es llamar la atención de los profesionales de la salud bucal sobre la necesidad de realizar un diagnóstico detallado para cada paciente a fin de proveer un registro estandarizado para todos los diagnósticos y facilitar la comparación de los datos a nivel internacional. Se espera que la CIE-OE contribuya en forma importante a la recolección de datos epidemiológicos sobre las enfemedades bucales menos frecuentes, para las cuales los métodos de vigilancia son impracticables. 